\title{
Killing vector fields and characteristic forms
}

\author{
By Xiang-Ming MEI \\ (Received September 13, 1993) \\ (Revised July 19, 1994)
}

\section{Introduction}

Let $(M, g)$ be a compact orientable Riemannian manifold, $\operatorname{dim} M=n=2 m$, then the Riemannian metric determines the Riemannian connection $\nabla$ uniquely. Let the corresponding connection form be $\omega$, then the curvature form is $\Omega=d \omega-\omega \wedge \omega$. The structure group of the tangent bundle $T(M)$ of $M$ is the special orthogonal group $S O(n)$, and the corresponding Lie algebra $\mathfrak{o}(n)$ is the algebra of real $(n \times n)$ skew symmetric matrices. Let $F$ be an $S O(n)$-invariant polynomial of degree $k$ defined on $\mathfrak{o}(n)$, then $F(\Omega)$ is a global closed $2 k$-form of $M$, so-called the characteristic form of $M$ defined by $F$. The purpose of this paper is to calculate the integral

$$
\int_{M} F(\Omega) \wedge \sigma
$$

where $\sigma$ is an arbitrary closed $(n-2 k)$-form of $M$.

When $\operatorname{deg} F=m$, this problem was solved by R. Bott [1] and P. BaumJ. Cheeger [2], but their method failed in the case $\operatorname{deg} F=k<m$. In this paper, we shall generalize their result to the case $k<m$, and give a formula for the above integral. All known results related to this problem, such as Pasternak [3], LazarovPasternak [4], Goméz [5] and the others are contained in our result as particular cases.

We assume that all geometric objects appearing in this paper are $C^{\infty}$, and we discuss in the real framework.

\section{The sets of singular points of the involutive vector fields}

In this paper, we fix the ranges of the indices as follows

$$
\begin{aligned}
A, B, C, \ldots & =1, \ldots, n \\
a, b, c, \ldots & =1, \ldots, 2 k \\
\alpha, \beta, \gamma, \ldots & =2 k+1, \ldots, n ; \\
\lambda, \mu, \nu, \ldots & =2 k, 2 k+1, \ldots, n .
\end{aligned}
$$


Given $(n-2 k+1) C^{\infty}$ vector fields $\left\{u_{2 k}, u_{2 k+1}, \ldots, u_{n}\right\}$ on the differentiable manifold $M, \operatorname{dim} M=n$, let $S$ be the set of singular points of $\left\{u_{\alpha}\right\}(\alpha=2 k+$ $1, \ldots, n)$ :

$S=\left\{x \in M:\right.$ the vectors $u_{2 k+1}(x), \ldots, u_{n}(x)$ are linearly dependent $\}$.

According to the results of Pontrjagin [6, Th.1], $S$ is the support of the dual cycle $D w_{2 k+1}$ of the $(2 k+1)$ th Stiefel-Whitney cocycle $w_{2 k+1}$ of $M$,

$$
\operatorname{dim} S \geq \operatorname{dim} D w_{2 k+1}=n-2 k-1 .
$$

Let $S(\varepsilon)$ be the $\varepsilon$-tubular neighbourhood of $S$ in $M$. On $M \backslash S(\varepsilon)$, the vector fields $\left\{u_{\alpha}\right\}$ are linearly independent everywhere. Let $N$ be the set of singular points of the vector fields $\left\{u_{2 k}, u_{2 k+1}, \ldots, u_{n}\right\}$ on $M \backslash S(\varepsilon)$ :

$$
\begin{aligned}
N=\{x \in M \backslash S(\varepsilon): & \text { the vectors } u_{2 k}(x), u_{2 k+1}(x), \ldots, u_{n}(x) \\
& \text { are linearly dependent }\},
\end{aligned}
$$

$N=\bigcup_{i=1}^{p} N_{i}$, where $N_{i}$ is the connected component of $N$. Denote $N_{i}(\varepsilon)$ to be the $\varepsilon$-tubular neighbourhood of $N_{i}$ in $M \backslash S(\varepsilon)$.

The vector fields $\left\{u_{\lambda}\right\}(\lambda=2 k, 2 k+1, \ldots, n)$ are called involutive, if there exists a set of $C^{\infty}$ functions $C_{\lambda \mu}{ }^{\nu}(\lambda, \mu, \nu=2 k, 2 k+1, \ldots, n)$ defined on $M$ such that

$$
\left[u_{\lambda}, u_{\mu}\right]=\sum_{\nu} C_{\lambda \mu}{ }^{\nu} u_{\nu}
$$

Lemma 1. For $\forall p \in N_{i}, u_{\lambda}(p) \in T_{p} N_{i}(\lambda=2 k, 2 k+1, \ldots, n)$; namely, the vectors $u_{\lambda}(p)$ are tangent to $N_{i}$.

Proof. We use the method of Baum [7] to prove this lemma, but state it in a different version.

Suppose $u_{2 k}(p) \notin T_{p} N_{i}$, there exists a coordinate chart $U_{p}$ centered at $p$, and the local coordinates $\left(x^{1}, \ldots, x^{n}\right)$ such that $u_{2 k}=\frac{\partial}{\partial x^{2 k}}$. Let $u_{\alpha}=\sum_{A} a_{\alpha}{ }^{A} \frac{\partial}{\partial x^{A}}$, where $a_{\alpha}{ }^{A}$ are $C^{\infty}$ functions defined on $U_{p}$, we define another set of the vector fields $u_{\alpha}^{\prime}$ as follows:

$$
u_{\alpha}^{\prime}=u_{\alpha}-a_{\alpha}^{2 k} \frac{\partial}{\partial x^{2 k}}=\sum_{A \neq 2 k} a_{\alpha}{ }^{A} \frac{\partial}{\partial x^{A}} .
$$

Since $u_{\alpha}^{\prime}=u_{\alpha}-a_{\alpha}^{2 k} u_{2 k}$, so that $u_{\alpha}^{\prime}$ and $u_{2 k}=\frac{\partial}{\partial x^{2 k}}$ are involutive,

$$
\left[u_{\alpha}^{\prime}, u_{\beta}^{\prime}\right]=\sum_{\gamma} C_{\alpha \beta}^{\prime}{ }^{\gamma} u_{\gamma}^{\prime}+C_{\alpha}^{\prime 2 k} \frac{\partial}{\partial x^{2 k}} .
$$


But there is no term $\frac{\partial}{\partial x^{2 k}}$ in $u_{\alpha}^{\prime}$, so that $\left[u_{\alpha}^{\prime}, u_{\beta}^{\prime}\right]$ doesn't involve the term $\frac{\partial}{\partial x^{2 k}}$, and therefore $C_{\alpha}^{\prime 2 k}=0$; it follows that the vector fields $\left\{u_{\alpha}^{\prime}\right\}$ are involutive. According to the Frobenius theorem, there exist a small coordinate chart $V \subset N_{p}$ and the local coordinates $\left(y^{1}, \ldots, y^{n}\right)$ such that $u_{\alpha}^{\prime}=\sum_{\beta} a_{\alpha}^{\prime} \frac{\partial}{\partial y^{\beta}}, u_{2 k}=\frac{\partial}{\partial x^{2 k}}=\frac{\partial}{\partial y^{2 k}}$. Consider the integral curve of the vector field $u_{2 k}$ in $V$ passing through $p$. It determines an one-parameter group of diffeomorphisms $f_{t}$. Let $x=f_{t}(p) \in V$, then since $u_{2 k} \notin T_{p} N_{i}, x \in V \backslash N_{i}$ unless $x=p$. If $x \in V, x \neq p, x$ is not a singular point, then the vectors $u_{2 k}(x)$ and $u_{\alpha}^{\prime}(x)$ are linearly independent. Define a diffeomorphism $g=$ $f_{t}^{-1}$ of $V$, then the tangential mapping $g_{*}: T_{x} M \rightarrow T_{p} M$ is a linear isomorphism. Since $g(x)=p$, so that the vectors $u_{2 k}(p)$ and $u_{\alpha}^{\prime}(p)$ or $u_{\alpha}(p)$ are also linearly independent, this means that $p$ is not a singular point of $\left\{u_{\lambda}\right\}$, contrary to the hypothesis, so that $u_{2 k}(p) \in T_{p} N_{i}$. The above proof is satisfied by any vector field linearly dependent on $\left\{u_{\alpha}\right\}$, so that $u_{\alpha}(p) \in T_{p} N_{i}$. The lemma is proved.

Corollary. For $\forall p \in N_{i}$, there exists a coordinate chart $U_{p}$ centered at $p$ with local coordinates $\left(x^{1}, \ldots, x^{n}\right)$ such that

$$
u_{\alpha}=\sum_{\beta} C_{\alpha}^{\beta} \frac{\partial}{\partial x^{\beta}}, \quad \text { on } \quad U_{p} \cap N_{i}
$$

where $C_{\alpha}^{\beta}$ are $C^{\infty}$ functions defined on $U_{p}$ and $\frac{\partial}{\partial x^{\beta}}$ are tangent to $N_{i}$.

Proof. According to the above lemma, the vector fields $\left\{u_{\lambda}\right\}(\lambda=2 k, 2 k+$ $1, \ldots, n)$ are tangent to $N_{i}$. Restrict them to $N_{i}$, then since the vector fields $\left\{u_{\lambda}\right\}$ are involutive, and $u_{2 k}$ is linearly dependent on $\left\{u_{\alpha}\right\}$ on $N_{i}$, the vector fields $\left\{u_{\alpha}\right\}$ are involutive on $N_{i}$. There exist linear combinations $\left\{u_{\alpha}^{\prime}\right\}$ of $\left\{u_{\alpha}\right\}$ such that $\left[u_{\alpha}^{\prime}, u_{\beta}^{\prime}\right]=0$, and according to the Frobenius theorem, there exist the local coordinates $\left(x^{1}, \ldots, x^{n}\right)$ in the coordinate chart $U_{p}$ such that $u_{\alpha}^{\prime}=\frac{\partial}{\partial x^{\alpha}}$ on $U_{p} \cap N_{i}$ and $\frac{\partial}{\partial x^{\beta}}=u_{\beta}^{\prime}=\sum_{\alpha} b_{\beta}^{\alpha} u_{\alpha}$, and it follows that $u_{\alpha}=\sum_{\beta} a_{\alpha}^{\beta} u_{\beta}^{\prime}=\sum_{\beta} a_{\alpha}^{\beta} \frac{\partial}{\partial x^{\beta}}$ where $\left(a_{\alpha}^{\beta}\right)=\left(b_{\beta}^{\alpha}\right)^{-1}$. Since $u_{\alpha}$ are tangent to $N_{i}, \frac{\partial}{\partial x^{\beta}}$ are also tangent to $N_{i}$.

\section{The actions of the Killing vector fields}

In the following, we use the notation $\left(v_{1}, \ldots, v_{p}\right)$ to denote the linear space spanned by the vectors $v_{1}, \ldots, v_{p}$.

Let $(M, g)$ be a compact orientable Riemannian manifold, $\operatorname{dim} M=n=2 m$, then the Riemannian metric $g$ determines uniquely the Riemannian connection $\nabla$ of the tangent bundle $T(M)$, and the connection form of $\nabla$ is $\omega$, and the corresponding curvature form is given by $\Omega=d \omega-\omega \wedge \omega$.

On a coordinate chart $U_{p}$ of $M$, let the coordinates be $\left(x^{1}, \ldots, x^{n}\right)$, and $\left\{\frac{\partial}{\partial x^{1}}, \ldots, \frac{\partial}{\partial x^{n}}\right\}$ the local frame of the tangent bundle $T(M)$ on $U_{p}$, then the matrix representation of the Riemannian metric, the Riemannian connection and the 
Riemannian curvature in $U_{p}$ are given by

$$
\begin{aligned}
& g=\left(g_{A B}\right), \quad g_{A B}=g_{B A}=g\left(\frac{\partial}{\partial x^{A}}, \frac{\partial}{\partial x^{B}}\right), \\
& g^{-1}=\left(g^{A B}\right), \\
& \nabla\left(\frac{\partial}{\partial x^{A}}\right)=\sum_{B} \omega_{A B} \frac{\partial}{\partial x^{B}}, \quad \omega=\left(\omega_{A B}\right), \\
& \omega_{A B}=\sum_{C} \Gamma_{A B}{ }^{C} \frac{\partial}{\partial x^{C}}, \quad \Gamma_{A B}^{C}=\frac{1}{2} \sum_{D} g^{C D}\left(\frac{\partial g_{A D}}{\partial x^{B}}+\frac{\partial g_{D B}}{\partial x^{A}}-\frac{\partial g_{A B}}{\partial x^{D}}\right), \\
& \Omega=\left(\Omega_{A B}\right), \quad \Omega_{A B}=d \omega_{A B}-\sum_{C} \omega_{A C} \wedge \omega_{C B} .
\end{aligned}
$$

The Riemannian connection keeps the Riemannian metric invariant; it means that $\nabla g=0$ or $\nabla_{Z} g=0$, for $\forall Z \in \Gamma(T(M))$, where $\nabla_{Z}=i(Z) \nabla$, in other words, for $\forall X, Y, Z \in \Gamma(T(M))$,

$$
Z . g(X, Y)=g\left(\nabla_{Z} X, Y\right)+g\left(X, \nabla_{Z} Y\right) .
$$

Let $\left\{u_{2 k}, u_{2 k+1}, \ldots, u_{n}\right\}$ be a set of involutive Killing vector fields; it means that they are involutive vector fields and also Killing vector fields, i.e. $L_{u_{\lambda}} g=0$ $(\lambda=2 k, 2 k+1, \ldots, n)$, where $L_{u_{\lambda}}$ means the Lie's derivative with respect to the vector field $u_{\lambda}$. In other words, for $\forall X, Y, Z \in \Gamma(T(M))$,

$$
u_{\lambda} \cdot g(X, Y)=g\left(L_{u_{\lambda}} X, Y\right)+g\left(X, L_{u_{\lambda}} Y\right)
$$

Let $F$ be an $S O(n)$-invariant polynomial of degree $k(k<m), F(\Omega)$ is a closed $2 k$-form of $M$. It is called the characteristic form of $M$ defined by $F$. Let $N_{i}$ be the connected component of the singular set $N$ of the involutive Killing vector fields $u=\left\{u_{\lambda}\right\}(\lambda=2 k, 2 k+1, \ldots, n)$, we define the residue of $u$ at the singular set $N_{i}$ to be

$$
\operatorname{Res}_{F}\left(u, N_{i}\right)=\int_{U} F(\Omega) \wedge \sigma
$$

where $U$ is an open set in $M \backslash S(\varepsilon)$ containing $N_{i}, U \supset N_{i}(\varepsilon) \supset N_{i}$ and $N_{i}$ is the deformation retract of $U$.

Consider the subbundle $E$ of $T(M)$; its fibre is defined to be

$$
E_{x}=\left(u_{2 k+1}(x), \ldots, u_{n}(x)\right)^{\perp}, \quad x \in U,
$$

where the notation " $\perp$ " means the orthogonal complement in the tangent space $T_{x}(M)$ with respect to the Riemannian metric $g$. 
The open set $U$ can be covered by the coordinate charts in the Lemma 1 , so that all problems in the following can be considered locally in $U_{p}$. Choose the local coordinates $\left(x^{1}, \ldots, x^{n}\right)$ in $U_{p}$ such that $\frac{\partial}{\partial x^{a}} \in \Gamma(E)$. Since $u_{\alpha}(x) \perp E_{x}$, so that

$$
0=g\left(\frac{\partial}{\partial x^{a}}, u_{\alpha}\right)=\sum_{\beta} a_{\alpha}^{\beta} g\left(\frac{\partial}{\partial x^{a}}, \frac{\partial}{\partial x^{\beta}}\right)=\sum_{\beta} a_{\alpha}^{\beta} g_{a \beta} .
$$

Since $\left\{u_{\alpha}\right\}$ are linearly independent on $U$, $\operatorname{det}\left(a_{\alpha}^{\beta}\right) \neq 0$, then $g_{a \beta}=0$. At the same time, $g^{a \beta}=0$.

$$
g=\left(\begin{array}{c|c}
g_{a b} & 0 \\
\hline 0 & g_{\alpha \beta}
\end{array}\right)=\left(\begin{array}{c|c}
g^{E} & 0 \\
\hline 0 & g_{\alpha \beta}
\end{array}\right)
$$

where $g^{E}=\left(g_{a b}\right)$ is the restriction of the Riemannian metric $g$ to the subbundle $E$.

Let $\nabla^{E}$ be the restriction of the Riemannian connection $\nabla$ to the subbundle $E$. On $U_{p}$ the matrix representation of the connection form $\omega^{E}$ and the curvature form $\Omega^{E}$ are given by

$$
\begin{aligned}
& \nabla^{E}\left(\frac{\partial}{\partial x^{a}}\right)=\sum_{b} \omega_{a b} \frac{\partial}{\partial x^{b}}, \\
& \omega^{E}=\left(\omega_{a b}\right), \quad \omega_{a b}=\sum_{A} \Gamma_{a}{ }^{b}{ }_{A} d x^{A}, \\
& \Omega^{E}=d \omega^{E}-\omega^{E} \wedge \omega^{E}=\left(\Omega_{a b}^{E}\right), \\
& \Omega_{a b}^{E}=d \omega_{a b}-\sum_{c} \omega_{a c} \wedge \omega_{c b} .
\end{aligned}
$$

LEMMA 2. In the coordinate chart $U_{p}$, the metric tensor $g_{a b}$ is a $C^{\infty}$ functions of the coordinates $x^{1}, \ldots, x^{2 k}$, and $\omega^{E}=\left(\omega_{a b}\right)$ is the Riemannian connection determined by the metric $g^{E}=\left(g_{a b}\right)$; it means that

$$
\omega_{a b}=\sum_{c} \Gamma_{a}{ }^{b}{ }_{c} d x^{c}, \quad \Gamma_{a}{ }^{b} c=\frac{1}{2} \sum_{d} g^{b d}\left(\frac{\partial g_{a d}}{\partial x^{c}}+\frac{\partial g_{c d}}{\partial x^{a}}-\frac{\partial g_{a c}}{\partial x^{d}}\right) .
$$

Proof. Since $\left\{u_{\alpha}\right\}(\alpha=2 k+1, \ldots, n)$ are Killing vector fields,

$$
\begin{aligned}
& u_{\alpha} \cdot g_{a b}=u_{\alpha} \cdot g\left(\frac{\partial}{\partial x^{a}}, \frac{\partial}{\partial x^{b}}\right)=g\left(\left[u_{\alpha}, \frac{\partial}{\partial x^{a}}\right], \frac{\partial}{\partial x^{b}}\right)+g\left(\frac{\partial}{\partial x^{a}},\left[u_{\alpha}, \frac{\partial}{\partial x^{b}}\right]\right), \\
& \sum_{\beta} a_{\alpha}^{\beta} \frac{\partial g_{a b}}{\partial x^{\beta}}=g\left(\left[\sum_{\beta} a_{\alpha}^{\beta} \frac{\partial}{\partial x^{\beta}}, \frac{\partial}{\partial x^{a}}\right], \frac{\partial}{\partial x^{b}}\right)+g\left(\frac{\partial}{\partial x^{a}},\left[\sum_{\beta} a_{\alpha}^{\beta} \frac{\partial}{\partial x^{\beta}}, \frac{\partial}{\partial x^{b}}\right]\right) .
\end{aligned}
$$


Note that $\frac{\partial}{\partial x^{\alpha}} \perp \frac{\partial}{\partial x^{a}}$ or $\frac{\partial}{\partial x^{b}}$, so

$$
\sum_{\beta} a_{\alpha}^{\beta} \frac{\partial g_{a b}}{\partial x^{\beta}}=\sum_{\beta} a_{\alpha}^{\beta}\left(g\left[\frac{\partial}{\partial x^{\beta}}, \frac{\partial}{\partial x^{a}}\right], \frac{\partial}{\partial x^{b}}\right)+g\left(\frac{\partial}{\partial x^{a}},\left[\frac{\partial}{\partial x^{\beta}}, \frac{\partial}{\partial x^{b}}\right]\right) .
$$

Since $\operatorname{det}\left(a_{\alpha}^{\beta}\right) \neq 0$, we have

$$
\begin{aligned}
& \frac{\partial g_{a b}}{\partial x^{\beta}}=0, \quad(a, b=1, \ldots, 2 k ; \beta=2 k+1, \ldots, n) . \\
& \omega_{a b}=\sum_{c} \Gamma_{a}{ }^{b}{ }_{c} d x^{c}+\sum_{\alpha} \Gamma_{a}{ }^{b}{ }_{\alpha} d x^{\alpha}
\end{aligned}
$$

where

$$
\begin{aligned}
& \Gamma_{a{ }^{b}}{ }^{b}=\frac{1}{2} \sum_{\alpha} g^{b d}\left(\frac{\partial g_{a d}}{\partial x^{\alpha}}+\frac{\partial g_{d \alpha}}{\partial x^{a}}+\frac{\partial g_{a \alpha}}{\partial x^{d}}\right)=0 \\
& \Gamma_{a c}{ }^{b}=\frac{1}{2} \sum_{d} g^{b d}\left(\frac{\partial g_{d c}}{\partial x^{a}}+\frac{\partial g_{a d}}{\partial x^{c}}-\frac{\partial g_{a c}}{\partial x^{d}}\right)=0 .
\end{aligned}
$$

So that the metric $g^{E}=\left(g_{a b}\right)$ determines the connection form $\omega^{E}=\left(\omega_{a b}\right)$ uniquely, in other words. $\nabla^{E}$ is the Riemannian connection of the metric $g^{E}$.

Corollary. On $U_{p}$, the curvature form $\omega^{E}$ is determined by $g^{E}$ uniquely.

Lemma 3.

$$
\operatorname{Res}_{F}\left(u, N_{i}\right)=\int_{U} F(\Omega) \wedge \sigma=\int_{U} F\left(\Omega^{E}\right) \wedge \sigma
$$

Proof. Since the structure group of the subbundle $E$ is the reduction of the structure group of the tangent bundle $T(M)$, we can extend the connection $\nabla^{E}$ of $E$ as the connection of $T(M)$ such that $\nabla^{E}\left(\frac{\partial}{\partial x^{\alpha}}\right)=0$.

Use the Chern-Weil technique [8] to construct the homotopy between the connections $\nabla$ and $\nabla^{E}$ of $T(M)$, let

$$
\begin{aligned}
& \omega_{t}=\omega^{E}+t u, \quad u=\omega-\omega^{E}, \\
& \Omega_{t}=d \omega_{t}-\omega_{t} \wedge \omega_{t},
\end{aligned}
$$

then there exists a $(2 k-1)$-form $\tau$ on $U_{p}$ such that

$$
F(\Omega)-F\left(\Omega^{E}\right)=d \tau,
$$

where

$$
\tau=k \int_{0}^{1} F(u, \underbrace{\Omega_{t}, \ldots, \Omega_{t}}_{(k-1)}) d t
$$




$$
\begin{aligned}
\int_{U} F(\Omega) \wedge \sigma & =\int_{U}\left(F\left(\Omega^{E}\right)+d \tau\right) \wedge \sigma=\int_{U} F\left(\Omega^{E}\right) \wedge \sigma+\int_{U} d(\tau \wedge \sigma) \\
& =\int_{U} F\left(\Omega^{E}\right) \wedge \sigma
\end{aligned}
$$

where $\int_{U} d(\tau \wedge \sigma)=\int_{\partial U} \tau \wedge \sigma=0$.

On $U \backslash N_{i}(\varepsilon)$, consider the action of the Killing vector fields $\left\{u_{\lambda}\right\}$ on the subbundle $E$. Let $v$ be the orthogonal projection of the Killing vector field $u_{2 k}$ on the subbundle $E$, i.e. $v=u_{2 k}-\sum_{\alpha} c_{\alpha} u_{\alpha}$, where $c_{\alpha}$ are the solutions of the linear equations $\sum_{\alpha} c_{\alpha} g\left(u_{\alpha}, u_{\beta}\right)=g\left(u_{2 k}, u_{\beta}\right)$. In the coordinate chart $U_{p}$, let $u_{2 k}=\sum_{A} v_{A} \frac{\partial}{\partial x^{A}}$. Since $\frac{\partial}{\partial x^{a}} \perp u_{\alpha}, v=\sum_{a} v_{a} \frac{\partial}{\partial x^{a}}$, where $v_{A}$ are $c^{\infty}$ functions on $U_{p}$. The vector field $v$ acts on the subbundle $E$ as follows: Let $X \in \Gamma(E)$,

$$
\begin{gathered}
v=\sum_{a} v_{a} \frac{\partial}{\partial x^{a}}, \quad X=\sum_{b} X_{b} \frac{\partial}{\partial x^{b}} \\
L_{v} X=[v, X]=\sum_{a, b}\left(v_{a} \frac{\partial X_{b}}{\partial x^{a}}-X_{a} \frac{\partial v_{b}}{\partial x^{a}}\right) \frac{\partial}{\partial x^{b}} \in \Gamma(E) .
\end{gathered}
$$

LEMma 4. On $U_{p} \backslash N_{i}(\varepsilon)$, the vector field $v$ is the Killing vector field of the Riemannian metric $g^{E}$, i.e. $L_{v} g^{E}=0$.

Proof. Since $u_{\lambda}$ is the Killing vector field of the Riemannian metric $g$, we have: For $\forall X, Y \in \Gamma(E)$,

$$
\begin{aligned}
u_{2 k} \cdot g(X, Y) & =g\left(\left[u_{2 k}, X\right], Y\right)+g\left(X,\left[u_{2 k}, Y\right]\right) \\
u_{\alpha} \cdot g(X, Y) & =g\left(\left[u_{\alpha}, X\right], Y\right)+g\left(X,\left[u_{\alpha}, Y\right]\right) .
\end{aligned}
$$

Since $X, Y \perp \frac{\partial}{\partial x^{\beta}}$, and $\operatorname{det}\left(a_{\alpha}^{\beta}\right) \neq 0$, it follows that

$$
\frac{\partial}{\partial x^{\beta}} g(X, Y)=g\left(\left[\frac{\partial}{\partial x^{\beta}}, X\right], Y\right)+g\left(X,\left[\frac{\partial}{\partial x^{\beta}}, Y\right]\right)
$$

but $\left[\frac{\partial}{\partial x^{\beta}}, X\right]$ and $\left[\frac{\partial}{\partial x^{\beta}}, Y\right] \in \Gamma(E)$, then we have

$$
\begin{aligned}
\frac{\partial}{\partial x^{\beta}} \cdot g^{E}(X, Y)= & g^{E}\left(\left[\frac{\partial}{\partial x^{\beta}}, X\right], Y\right)+g^{E}\left(X,\left[\frac{\partial}{\partial x^{\beta}}, Y\right]\right), \\
v \cdot g^{E}(X, Y)= & \left(u_{2 k}-\sum_{\alpha} v_{\alpha} \frac{\partial}{\partial x^{\alpha}}\right) \cdot g(X, Y) \\
= & g\left(\left[u_{2 k}, X\right], Y\right)+g\left(X,\left[u_{2 k}, Y\right]\right) \\
& -\sum_{\alpha} v_{\alpha} g\left(\left[\frac{\partial}{\partial x^{\alpha}}, X\right], Y\right)+g\left(X,\left[\frac{\partial}{\partial x^{\alpha}}, Y\right]\right),
\end{aligned}
$$


Since $X, Y \perp \frac{\partial}{\partial x^{\alpha}}$, we have

$$
\begin{aligned}
v \cdot g^{E}(X, Y)= & g\left(\left[u_{2 k}, X\right], Y\right)+g\left(X,\left[u_{2 k} . Y\right]\right)-g\left(\left[\sum_{\alpha} v_{\alpha} \frac{\partial}{\partial x^{\alpha}}, X\right], Y\right) \\
& -g\left(X,\left[\sum_{\alpha} v_{\alpha} \frac{\partial}{\partial x^{\alpha}}, Y\right]\right)=g([v, X], Y)+g(X,[v, Y]) \\
= & g^{E}([v, X], Y)+g^{E}(X,[v, Y])
\end{aligned}
$$

it follows that $L_{v} g^{E}=0$.

Note that $\nabla^{E}$ is the Riemannian connection determined uniquely by the metric $g^{E}$, according to Kobayashi-Nomizu ([9], p.237), the infinitesmal isometry must be an infinitesmal affine transformation, we can deduce from $L_{v} g^{E}=0$ that $L_{v} \omega^{E}=0$, and thus we have

Lemma 5. On $U \backslash N_{i}(\varepsilon)$, for $\forall Y \in \Gamma(E)$, the following formula holds

$$
L_{v} \cdot \nabla_{Y}^{E}-\nabla_{Y}^{E} \cdot L_{v}-\nabla_{[v, Y]}^{E}=0 .
$$

Proof. Since $L_{v} \omega^{E}=0$, we have

$$
\begin{aligned}
0 & =L_{v} \omega^{E}(Y)=i(v) d \omega^{E}(Y)+d\left(i(v) \omega^{E}\right)(Y) \\
& =d \omega^{E}(v, Y)+\left(d \omega^{E}(v)\right)(Y) \\
& =v \cdot \omega^{E}(Y)-Y \cdot \omega^{E}(v)-\omega^{E}([v, Y])+Y \cdot \omega^{E}(v),
\end{aligned}
$$

and then

$$
v \cdot \omega^{E}(Y)-\omega^{E}([v, Y])=0 .
$$

On the other hand, for $\forall Z \in \Gamma(E)$,

$$
\begin{aligned}
L_{v}( & \left.\nabla_{Y}^{E} Z\right)-\nabla_{Y}^{E}\left(L_{v} Z\right)-\nabla_{[v, Y]}^{E} Z \\
= & L_{v}\left(Y . Z+\omega^{E}(Y) Z\right)-Y .[v, Z]-\omega^{E}(Y)[v, Z]-[v, Y] . Z-\omega^{E}([v, Y])(Z) \\
= & {[v, Y . Z]+v \cdot \omega^{E}(Y) Z+\omega^{E}(Y)[v, Y]-Y . v . Z+Y . Z . v } \\
& -\omega^{E}(Y)[v \cdot Z]-v \cdot Y . Z+Y \cdot v \cdot Z-\omega^{E}([v, Y])(Z) \\
= & \left(v \cdot \omega^{E}(Y)-\omega^{E}([v, Y])\right)(Z)=0 .
\end{aligned}
$$

Now we introduce a new connection $\widetilde{\nabla}$ in the subbundle $E$ as follows:

$$
\begin{aligned}
& \widetilde{\nabla}_{v}=L_{v}, \\
& \widetilde{\nabla}_{Y}=\nabla_{Y}^{E}, \text { if } g(v \cdot Y)=0 .
\end{aligned}
$$


Let the connection form of $\widetilde{\nabla}$ be $\widetilde{\omega}$, and the corresponding curvature form be $\widetilde{\Omega}=$ $d \widetilde{\omega}-\widetilde{\omega} \wedge \widetilde{\omega}$, then we have

Theorem 1. (Vanishing theorem) On $U \backslash N_{i}(\varepsilon)$,

$$
F(\widetilde{\Omega})=0 .
$$

Proof. For $\forall Y_{r} \in \Gamma(E)(r=1, \ldots, 2 k-1), Y_{r} \perp v$,

$$
\begin{aligned}
& \widetilde{\Omega}(v \cdot Y)=\widetilde{\nabla}_{v} \widetilde{\nabla}_{Y}-\widetilde{\nabla}_{Y} \widetilde{\nabla}_{v}-\widetilde{\nabla}_{[v, Y]}=L_{v} \cdot \nabla_{Y}^{E}-\nabla_{Y}^{E} \cdot L_{v}-\nabla_{[v, Y]}^{E}=0, \\
& F(\widetilde{\Omega})\left(Y_{1}, \ldots, Y_{2 k-1}, v\right)=F\left(\widetilde{\Omega}\left(Y_{1}, Y_{2}\right), \ldots, \widetilde{\Omega}\left(Y_{2 k-1}, v\right)\right)=0,
\end{aligned}
$$

and the theorem is proved.

Corollary. There exists a $(2 k-1)$-form $\eta$ on $U \backslash N_{i}(\varepsilon)$ such that

$$
F\left(\Omega^{E}\right)=-d \eta
$$

Proof. Construct the homotopy between the connections $\nabla^{E}$ and $\widetilde{\nabla}$. Let

$$
\begin{aligned}
& \omega_{t}=\omega^{E}+t u, \quad u=\widetilde{\omega}-\omega^{E}, \\
& \Omega_{t}=d \omega_{t}-\omega_{t} \wedge \omega_{t},
\end{aligned}
$$

according to the Chern-Weil technique

$$
\eta=k \int_{0}^{1} F(u, \underbrace{\Omega_{t}, \ldots, \Omega_{t}}_{(k-1)}) d t .
$$

In the following, we shall give an explicit formula for the transgressive form $\eta$. Define a 1-form $\pi$ on $U \backslash N_{i}(\varepsilon)$ as follows: For $\forall X \in \Gamma(T(M))$,

$$
\pi(X)=\frac{g(v, X)}{g(v, v)},
$$

then $\pi(v)=1$ and $\pi(Y)=0$, if $g(v, Y)=0$.

Consider an operator $A_{v}=L_{v}-\nabla_{v}^{E}$ on the subbundle $E . A_{v}(x)$ is a linear transformation on the fibre $E_{x}$. For $\forall X \in \Gamma(T(M))$,

$$
u(X) \frac{\partial}{\partial x^{a}}=\widetilde{\omega}(X) \frac{\partial}{\partial x^{a}}-\omega^{E}(X) \frac{\partial}{\partial x^{a}}=\left(\widetilde{\nabla}_{X}-\nabla_{X}^{E}\right) \frac{\partial}{\partial x^{a}},
$$

when $X=v$,

$$
u(v)=\left(\widetilde{\nabla}_{v}-\nabla_{v}^{E}\right)=\left(L_{v}-\nabla_{v}^{E}\right)=A_{v}=\pi(v) \otimes A_{v} .
$$


If $Y \in \Gamma(T(M)), Y \perp v$, then

$$
u(Y)=\widetilde{\nabla}_{Y}-\nabla_{Y}^{E}=0=\pi(Y) \otimes A_{v} .
$$

So we have

$$
\begin{aligned}
& u=\pi \otimes A_{v} . \\
& \omega_{t}=\omega^{E}+t u=\omega^{E}+t \pi \otimes A_{v}, \\
& \Omega_{t}=d \omega_{t}-\omega_{t} \wedge \omega_{t}=\Omega^{E}+t d \pi \otimes A_{v}(\bmod . \pi), \\
& \eta=k \int_{0}^{1} F(u, \Omega_{t}, \underbrace{\ldots,}_{(k-1)} \Omega_{t}) d t=k \int_{0}^{1} F(\pi \otimes A_{v}, \Omega^{E}+t d \underbrace{\pi \otimes A_{v}, \ldots}_{(k-1)}) d t \\
&= \sum_{p=0}^{k-1} C_{k}^{p} F(\Omega^{E} \underbrace{\ldots, \ldots}_{p} \Omega^{E}, A_{v} \underbrace{\ldots \ldots}_{(k-p-1)} A_{v}) \wedge \pi \wedge(d \pi)^{k-p-1} \\
&= \text { The coefficient of } t^{k-1} \text { in the Taylor expansion of the } \\
& \quad \text { expression }\left\{F\left(t \Omega^{E}+A_{v}\right) \wedge \frac{\pi}{1-t d \pi}\right\} .
\end{aligned}
$$

\section{The residue of the singular set of the involutive Killing vector fields}

Let $(M, g)$ be a compact orientable Riemannian manifold, $\operatorname{dim} M=n=$ $2 m$, and $u=\left\{u_{2 k}, u_{2 k+1}, \ldots, u_{n}\right\}$ be a set of involutive Killing vector fields defined on $M . S$ is the singular set of $\left\{u_{2 k+1}, \ldots, u_{n}\right\}, N$ is the singular set of $\left\{u_{2 k}, u_{2 k+1}, \ldots, u_{n}\right\}$ in $M \backslash S(\varepsilon), N=U_{i} N_{i}$, where $N_{i}$ 's are connected components of $M$. Let $N_{i}(\varepsilon)$ be the $\varepsilon$-tubular neighbourhood of $N_{i}$ in $M \backslash S(\varepsilon)$, and $U$ be an open set in $M \backslash S(\varepsilon)$ containing $N_{i}, U \supset N_{i}(\varepsilon) \supset N_{i}, N_{i}$ being the deformation retract of $U$. Note that $U$ can be covered by the coordinate charts $U_{p}$ in Lemma 1 , so we can discuss our problem locally in $U_{p}$, then extend the results to whole $U$ using the technique of partition of unity. In the following, we want to calculate the residue $\operatorname{Res}_{F}\left(u, N_{i}\right)$.

Let $E$ be a subbundle of the tangent bundle $T(M)$ on $U \backslash S(\varepsilon)$, with its fibre defined by

$$
E_{x}=\left(u_{2 k+1}(x), \ldots, u_{n}(x)\right)^{\perp}, \quad x \in U \backslash S(\varepsilon) .
$$

Let $v$ be the orthogonal projection of the Killing vector field $u_{2 k}$ on the subbundle $E$, then $N_{i}$ is the zero set of $v$. The operator $A_{v}=L_{v}-\nabla_{v}^{E}$ acts on the subbundle $E, A_{v}(x)$ can be considered as a linear transformation of the fibre $E_{x}$. For $\forall X$, $Y \in \Gamma(E)$

$$
\begin{aligned}
& v \cdot g^{E}(X, Y)=g^{E}\left(L_{v} X, Y\right)+g^{E}\left(X, L_{v} Y\right) \\
& v \cdot g^{E}(X, Y)=g^{E}\left(\nabla_{v}^{E} X, Y\right)+g^{E}\left(X, \nabla_{v}^{E} Y\right) .
\end{aligned}
$$


Subtract these two expressions, we then get

$$
g^{E}\left(A_{v} X, Y\right)+g^{E}\left(X, A_{v} Y\right)=0
$$

so that the linear transformation $A_{v}(x)$ in the fibre $E_{x}$ is skew symmetric with respect to the metric $g^{E}$.

On $N_{i}$, although $v=0$, the linear transformation $A_{v}(x)$ is still meaningful. Since $A_{v}(x)$ is skew symmetric, the linear transformation $A_{v}(x)$ in the fibre $E_{x}$ can be reduced to the following canonical form:

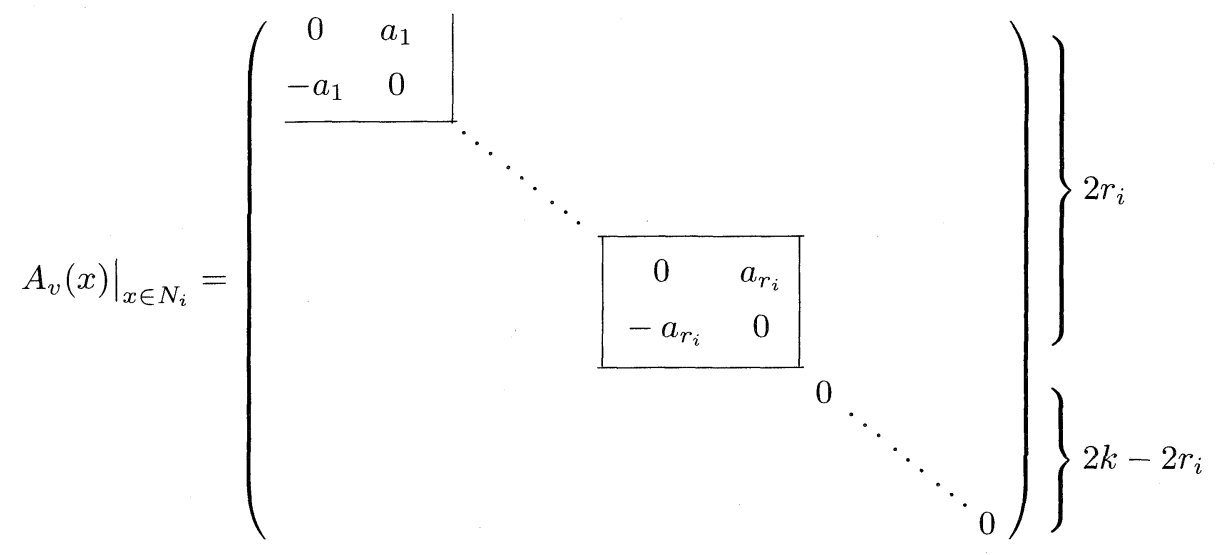

Note that $\nabla^{E}$ is the Riemannian connection determined by $g^{E}$, it has no torsion, i.e.

$$
\nabla_{v}^{E} X-\nabla_{X}^{E} v=L_{v} X
$$

so that $A_{v} X=-\nabla_{X}^{E} v$. Let $X \in E_{x} \cap T_{x}\left(N_{i}\right)$, extend it as a vector field on $N_{i}$, the integral curve of this vector field passing through $x$ is contained in $N_{i}$, and the vector field $v=0$ along this curve, hence $\nabla_{X}^{E} v=0$. It means that: When $X \in E_{x} \cap T_{x}\left(N_{i}\right), A_{v}(x)(X)=0$.

Consider a subbundle $T^{\perp}\left(N_{i}\right)$ of the tangent bundle $T(M)$ on $N_{i}$, its fibre $T_{x}^{\perp}\left(N_{i}\right)$ is defined as $\left(T_{x}\left(N_{i}\right)\right)^{\perp}$. According to the Lemma $1,\left(u_{2 k+1}(x), \ldots\right.$, $\left.u_{n}(x)\right) \subset T_{x}\left(N_{i}\right)$, so that $T_{x}^{\perp}\left(N_{i}\right) \subset E_{x}=\left(u_{2 k+1}(x), \ldots, u_{n}(x)\right)^{\perp}$, it means that $T^{\perp}\left(N_{i}\right)$ is the subbundle of $E$ on $N_{i}$.

When $X \in T_{x}^{\perp} N_{i}$, locally $X(x)=\sum_{a} X^{a}(x) \frac{\partial}{\partial x^{a}}, X^{a}(x) \neq 0$.

$$
\begin{aligned}
A_{v} X(x) & =-\nabla_{X}^{E} v(x)=-\left(d v(X)+\omega^{E}(X) \cdot v\right)(x) \\
& =-d v(X)(x)=-\sum_{a} X^{a}(x) \frac{\partial v}{\partial x^{a}}(x) .
\end{aligned}
$$

If the points of $N_{i}$ are non-degenerate zeros of the vector field $v$, i.e. $\operatorname{det}\left(\frac{\partial v^{b}}{\partial x^{a}}(x)\right) \neq$ 0 , then $A_{v} X(x) \neq 0$. This fact tells us: $\operatorname{dim} T_{x}^{\perp}\left(N_{i}\right)=2 r_{i}$. We have also 
LEMma 6. If $N_{i}$ is the set of non-degenerate zeros of the vector field $v$, then $\operatorname{dim} N_{i}=n-2 r_{i}=2\left(m-r_{i}\right)$.

Proof. $\operatorname{dim} N_{i}=\operatorname{dim} T_{x}\left(N_{i}\right)=n-\operatorname{dim} T_{x}^{\perp}\left(N_{i}\right)=n-2 r_{i}$.

Let the induced connection of the connection $\nabla^{E}$ of the bundle $E$ to the subbundle $T^{\perp}\left(N_{i}\right)$ be $\nabla^{\perp}$, and the corresponding curvature form be $\Omega^{\perp}$. Define the operator $A_{v}^{\perp}$ as the restriction of the operator $A_{v}$ to $T^{\perp}\left(N_{i}\right)$. Now we can prove our main result as follows:

THEOREM 2. If $N_{i}$ is the set of non-degenerate zeros of the vector field $v$, then

$$
\begin{aligned}
\operatorname{Res}_{F}\left(u, N_{i}\right) & =\int_{U} F(\Omega) \\
& =\int_{N_{i}}\left\{\text { The coefficient of } t^{k-r_{i}}\right. \text { in the Taylor expansion } \\
& \text { of the expression } \left.\frac{F\left(t \Omega^{E}+A_{v}\right)}{E\left(t \Omega^{\perp}+A_{v}^{\perp}\right)}\right\} \wedge \sigma,
\end{aligned}
$$

where $E\left(\Omega^{\perp}\right)$ is the Euler characteristic form of the vector bundle $T^{\perp}\left(N_{i}\right)$, and $\sigma$ is any closed $(n-2 k)$-form defined on $N_{i}$.

Proof. According to Lemma 3, we have

$$
\begin{aligned}
\int_{U} F(\Omega) \wedge \sigma & =\int_{U} F\left(\Omega^{E}\right) \wedge \sigma=\lim _{\varepsilon \rightarrow 0} \int_{U \backslash N_{i}(\varepsilon)}-d \eta \wedge \sigma \\
& =\lim _{\varepsilon \rightarrow 0} \int_{\partial N_{i}(\varepsilon)} \eta \wedge \sigma,
\end{aligned}
$$

Consider the $\varepsilon$-tubular neighbourhood $N_{i}(\varepsilon)$ as a sphere bundle on $N_{i}$, its fibre is an $\left(n-2 r_{i}-1\right)$-dimensional sphere $S_{i}(\varepsilon)$. According to the corollary of Theorem 1 ,

$$
\begin{aligned}
& \int_{U} F(\Omega) \wedge \sigma=\lim _{\varepsilon \rightarrow 0} \int_{N_{i}(\varepsilon)}\left\{\text { The coefficient of } t^{k-1}\right. \text { in the Taylor } \\
&\text { expansion of the expression } \left.F\left(t \Omega^{E}+A_{v}\right) \wedge \frac{\pi}{1-t d \pi}\right\} \wedge \sigma
\end{aligned}
$$

For the integral in the parenthesis, we have

\section{Lemma 7.}

$$
\begin{aligned}
\lim _{\varepsilon \rightarrow 0} \int_{S_{i}(\varepsilon)} \frac{\pi}{1-t d \pi}= & \text { The coefficient of } t^{r_{i}-1} \text { in the Taylor } \\
& \text { expansion of the expression } \frac{1}{E\left(t \Omega^{\perp}+A_{v}^{\perp}\right)} .
\end{aligned}
$$


Proof. See Kobayashi [10, pp.72-75].

According to the above lemma, we have

$$
\begin{aligned}
\int_{U} F(\Omega) \wedge \sigma=\int_{N_{i}}\left\{\text { The coefficient } t^{(k-1)-\left(r_{i}-1\right)}\right. \text { in the } \\
\text { Taylor expansion of the expression } \left.\frac{F\left(t \Omega^{E}+A_{v}\right)}{E\left(t \Omega^{\perp}+A_{v}^{\perp}\right)}\right\} \wedge \sigma .
\end{aligned}
$$

The theorem is proved.

\section{The integral formula of the characteristic form}

Let $(M, g)$ be a compact orientable Riemannian manifold, $\operatorname{dim} M=n=2 m$, and $u=\left\{u_{2 k}, u_{2 k+1}, \ldots, u_{n}\right\}$ be a set of involutive Killing vector fields defined on $M$. Let $S$ be the singular set of the vector fields $\left\{u_{2 k+1}, \ldots, u_{n}\right\}$, we suppose that they are situated in general position, then $\operatorname{dim} S=n-2 k-1$.

Note that the subbundle $E$ is undefined on $S$, and $F\left(\Omega^{E}\right)$ is also undefined on $S$. But if the closed $(n-2 k)$-form $\sigma$ of $M$ vanishes on $S$, then $F\left(\Omega^{E}\right) \wedge \sigma$ is still meaningful on $S$. following:

In this section, we want to calculate the integral $\int_{M} F(\Omega) \wedge \sigma$. We need the

LEMMA 8. There exists another closed $(n-2 k)$-form $\sigma^{\prime}$ on $M$ and a sufficiently small $\varepsilon$ such that $\sigma^{\prime} \sim \sigma$ and $\sigma^{\prime}=0$ on the closure of the tubular neighbourhood $S\left(\frac{1}{4} \varepsilon\right) ; \sigma^{\prime}=\sigma$ on $M \backslash S\left(\frac{3}{4} \varepsilon\right)$.

Proof. Since $\sigma$ is a closed $(n-2 k)$-form of $M$ and $\operatorname{dim} S=n-2 k-1, \sigma \mid S=0$. For a sufficient small $\varepsilon$, the tubular neighbourhood $S(\varepsilon)$ of $S$ is the deformation retract of $S$. Let $\pi: S(\varepsilon) \rightarrow S$ be the projection, then $\sigma \mid S(\varepsilon) \sim \pi^{*}(\sigma \mid S)$, so that $\sigma \mid S(\varepsilon) \sim 0$., it means that: there exists a $(n-2 k-1)$-form $\theta$ on $S(\varepsilon)$ such that $\sigma \mid S(\varepsilon)=d \theta$. Extend $\theta$ as a form of $M$. Constructs a $C^{\infty}$ function $\phi$ on $M$ such that $\left.\phi\right|_{S\left(\frac{1}{4} \varepsilon\right)}=1,\left.\phi\right|_{M \backslash S\left(\frac{3}{4} \varepsilon\right)}=0$, then we define $\sigma^{\prime}=\sigma-d(\phi \theta)$. This closed form satisfies the requirement of the lemma.

Corollary.

$$
\int_{M} F(\Omega) \wedge \sigma=\int_{M} F(\Omega) \wedge \sigma^{\prime}=\int_{M} F\left(\Omega^{E}\right) \wedge \sigma^{\prime}
$$

ProOF.

$$
\int_{M} F(\Omega) \wedge \sigma=\int_{M} F(\Omega) \wedge\left(\sigma^{\prime}+d(\phi \theta)\right)
$$




$$
\begin{aligned}
& =\int_{M} F(\Omega) \wedge \sigma^{\prime}+\int_{M} d(\phi \theta) \\
& =\int_{M} F(\Omega) \wedge \sigma^{\prime}=\lim _{\varepsilon \rightarrow 0} \int_{M \backslash S_{i}(\varepsilon)} F(\Omega) \wedge \sigma^{\prime} \\
& =\lim _{\varepsilon \rightarrow 0} \int_{M \backslash S_{i}(\varepsilon)}\left(F\left(\Omega^{E}\right)+d \tau\right) \wedge \sigma^{\prime} .
\end{aligned}
$$

When $\varepsilon \rightarrow 0,\left.\sigma^{\prime}\right|_{S_{i}(\varepsilon)}=0$, so that

$$
\begin{aligned}
\int F(\Omega) \wedge \sigma^{\prime} & =\lim _{\varepsilon \rightarrow 0} \int_{M \backslash S_{i}(\varepsilon)}\left(F\left(\Omega^{E}\right)+d \tau\right) \wedge \sigma^{\prime} \\
& =\int_{M}\left(F\left(\Omega^{E}\right)+d \tau\right) \wedge \sigma^{\prime}=\int_{M} F\left(\Omega^{E}\right) \wedge \sigma^{\prime}+\int_{M} d\left(\tau \wedge \sigma^{\prime}\right) \\
& =\int_{M} F\left(\Omega^{E}\right) \wedge \sigma^{\prime}
\end{aligned}
$$

We obtain the integral formula of the general characteristic form as follows:

Theorem 3.

$$
\int_{M} F(\Omega) \wedge \sigma=\sum_{i=1}^{p} \operatorname{Res}_{F}\left(u, N_{i}\right) .
$$

Proof. According to the above corollary,

$$
\begin{aligned}
\int_{M} F(\Omega) \wedge \sigma & =\int_{M} F\left(\Omega^{E}\right) \wedge \sigma^{\prime}=\lim _{\varepsilon \rightarrow 0} \int_{M \backslash \bigcup_{i=1}^{p} N_{i}(\varepsilon)}-d \eta \wedge \sigma^{\prime} \\
& =\sum_{i=1}^{p} \lim _{\varepsilon \rightarrow 0} \int_{\partial N_{i}(\varepsilon)} \eta \wedge \sigma^{\prime},
\end{aligned}
$$

Since $\partial N_{i}(\varepsilon) \cap S_{i}(\varepsilon)=\phi$, in the above integral, $\sigma^{\prime}=\sigma$.

It follows from the proof of Theorem 2 that:

$$
\begin{aligned}
\int_{M} F(\Omega) \wedge \sigma & =\sum_{i=1}^{p} \lim _{\varepsilon \rightarrow 0} \int_{\partial N_{i}(\varepsilon)} \eta \wedge \sigma=\sum_{i=1}^{p} \int_{U_{i}} F\left(\Omega^{E}\right) \wedge \sigma \\
& =\sum_{i=1}^{p} \lim _{\varepsilon \rightarrow 0} \int_{U_{i}} F(\Omega) \wedge \sigma=\sum_{i=1}^{p} \operatorname{Res}_{F}\left(u, N_{i}\right) .
\end{aligned}
$$

The theorem is proved.

In the following, we shall show that: many known results are the particular cases of the above theorem. 
(1) When $k=m$, then the Riemannian manifold $(M, g)$ has only one Killing vector field $u$. In this case, the subbundle $E$ coincides with the tangent bundle $T(M)$.

(a) If the singular points $N_{i}(i=1, \ldots, p)$ of the Killing vector field $u$ are isolated, then $\operatorname{dim} N_{i}=0, r_{i}=k=m$, so that the subbundle $T^{\perp}\left(N_{i}\right)$ also coincides with $T(M), E\left(\Omega^{\perp}\right)=E(\Omega)=\sqrt{\operatorname{det} \Omega}$, so that

$$
\int_{M} F(\Omega)=\sum_{i=1}^{p} \operatorname{Res}_{F}\left(u, N_{i}\right)=\sum_{i=1}^{p} \frac{F\left(A_{v}\left(N_{i}\right)\right)}{\sqrt{\operatorname{det} A_{v}\left(N_{i}\right)}} .
$$

This is the result of Bott [1].

(b) If the singular points of $u$ are non-isolated, let $N_{i}(i=1, \ldots, p)$ be the connected components of the singular sets of $u$, and $\operatorname{dim} N_{i}=n-2 r_{i}$, then $T^{\perp}\left(N_{i}\right)$ is a subbundle of the tangent bundle $T(M)$.

$$
\begin{aligned}
\int_{M} F(\Omega) & =\sum_{i=1}^{p} \operatorname{Res}_{F}\left(u, N_{i}\right) \\
= & \sum_{i=1}^{p} \int_{N_{i}}\left\{\text { The coefficient of } t^{m-r_{i}}\right. \text { in the Taylor expansion } \\
& \text { of the expression } \left.\frac{F\left(t \Omega+A_{v}\right)}{E\left(t \Omega^{\perp}+A_{v}^{\perp}\right)}\right\} .
\end{aligned}
$$

This is the result of Baum-Cheeger [2].

(2) When $k<m$, but the involutive Killing vector fields $u=\left\{u_{2 k}, u_{2 k+1}, \ldots\right.$, $\left.u_{n}\right\}$ are situated in general position, then $\operatorname{dim} N=\operatorname{dim} N_{i}=n-2 k=2(m-k)$ and $r_{i}=k$, so that the subbundle $T^{\perp}\left(N_{i}\right)$ coincides with $E$,

$$
\begin{aligned}
\int_{M} F(\Omega) \wedge \sigma & =\sum_{i=1}^{p} \operatorname{Res}_{F}\left(u, N_{i}\right) \\
& =\sum_{i=1}^{p} \int_{N_{i}}\{\text { The constant term in the Taylor expansion of } \\
\text { the expression } \left.\frac{F\left(t \Omega^{E}+A_{v}\right)}{E\left(t \Omega^{E}+A_{v}\right)}\right\} \wedge \sigma & \\
= & \sum_{i=1}^{p} \int_{N_{i}}\left(\frac{F\left(A_{v}\right)}{\sqrt{\operatorname{det} A_{v}}}\right) \sigma
\end{aligned}
$$

This is the result of Lazarov-Pasternak [4].

(3) Let $M$ be a compact orientable differentiable manifold, $\operatorname{dim} M=n=2 m$, with a $(n-2 k+1)$-dimensional compact Lie group $G$ acting on it. We can introduce 
a $G$-invariant metric $g$ in $M$, then $M$ becomes a Riemannian manifold. The basis of the Lie algebra of $G\left\{X_{\lambda}\right\}(\lambda=2 k, 2 k+1, \ldots, n)$ induces a set of involutive Killing vector fields $\left\{u_{\lambda}\right\}$ on the Riemannian manifold $(M, g)$. Then we obtain the result of Pasternak [3] from Theorem 3.

If the Lie group $G$ acting on $M$ is Abelian, then $\left[X_{\lambda}, X_{\mu}\right]=0$, so we obtain the result of Goméz [5].

\title{
References
}

[ 1 ] R. Bott, A residue formula for holomorphic vector fields, J. Differential Geometry, 1 (1967), 311-330.

[2] P.F. Baum and J. Cheeger, Infinitesmal isometries and Pontrjagin numbers, Topology, 8 (1969), 173-193.

[ 3 ] J. Pasternak, Foliations and compact Lie group actions, Comment. Math. Helv., 46 (1971), 467-477.

[ 4 ] C. Lazarov and J. Pasternak, Residues and characteristic classes for Riemannian foliations, J. Differential Geometry, 11 (1976), 599-612.

[ 5 ] F. Goméz, A residue formula for characteristic classes, Topology, 21 (1982), 101-124.

[6] L.S. Pontrjagin, Vector fields on manifolds, Math. Sbornik (N.S.), 24 (1949), 129-162, (in Russian).

[ 7 ] P.F. Baum, Structure of foliation singularities, Adv. in Math., 15 (1975), 361-374.

[ 8 ] S.S. Chern, Geometry of characteristic classes, Proc. 13th, Biennar Siminar, (1972), 1-40.

[ 9 ] S. Kobayashi and K. Nomizu, Foundations of Differential Geometry, John Wiley \& Sons, New York, 1963.

[10] S. Kobayashi, Transformation Groups in Differential Geometry, Springer-Verlag, Berlin, 1972 .

\author{
Capital Normal University \\ BEIJING, 100037 \\ P. R. OF CHINA
}

